\title{
Tunable dynamics of a flake on graphene: Libration frequency
}

\author{
O. Üzengi Aktürk, ${ }^{1,2,3}$ E. Aktürk, ${ }^{1,2,4,{ }^{*}}$ H. H. Gürel, ${ }^{5}$ and S. Ciraci ${ }^{2, \dagger}$ \\ ${ }^{1}$ Nanotechnology Application and Research Center, Adnan Menderes University, Aydin 09010, Turkey \\ ${ }^{2}$ Department of Physics, Bilkent University, 06800 Ankara, Turkey \\ ${ }^{3}$ Department of Electric and Electronic Engineering, Adnan Menderes University, 09100, Aydin, Turkey \\ ${ }^{4}$ Department of Physics, Adnan Menderes University, 09100, Aydin, Turkey \\ ${ }^{5}$ Department of Information Systems Engineering, Kocaeli University, 41380 Kocaeli, Turkey \\ (Received 7 April 2016; revised manuscript received 13 February 2017; published 9 March 2017)
}

\begin{abstract}
In this paper we investigated the interaction between a graphene nanoflake anchored to the 2D graphene monolayer. This interaction is attractive but weak and is capable of setting a well defined registry in equilibrium. Rotational and linear displacements from equilibrium registry generate restoring forces, which can be controlled by external agents. Similar flakes can be self-assembled and can also execute simple harmonic motion as if a physical pendulum. Oscillation of a nanoflake about their equilibrium registries resulting in a characteristic libration frequency is predicted. This frequency depends on the size and geometry of the flake. Moreover, the libration frequency, as well as the electronic and magnetic properties of the flake+monolayer systems, can be tuned by a foreign molecule anchored to the flake, by electric charging and applied parallel and perpendicular electric and magnetic fields. When the sliding of the flake is combined with rotation, the friction force can be reduced dramatically. It is surprising that weak interaction can offer such features at nanoscale, which may offer potential applications. Our predictions are obtained by first-principles calculations based on density functional theory.
\end{abstract}

DOI: 10.1103/PhysRevB.95.125413

\section{INTRODUCTION}

The interaction between molecules or nanoflakes anchored on the monolayer (ML) structures, such as graphene, h$\mathrm{BN}, \mathrm{h}-\mathrm{MoS}_{2}$, etc. or their thin films, is weak and has predominantly van der Waals and partly chemical character [1]. Since molecular NEMs and nanomechanical devices have shown potential applications in future technologies [2-4], the rotational and translational dynamics of these nanoflakes, in particular graphene flakes ("nano" will be omitted in the rest of the text) and molecules have been brought into focus [2,5-9]. Like an atom switch [10], these single molecules have been shown to execute controlled rotation or translation through the electrical bias exerted by the tip of a scanning tunneling microscope $[3,4,11,12]$. Additionally, the configurations of the molecules, as well as their dynamics on the monolayer structures, have been imaged clearly by STM [13-16].

Even if molecules or flakes anchored on 2D ML structures can maintain a well-defined registry in their equilibrium state, it may be rather easy to rotate or slide them relative to each other, since the energy barrier to rotation $Q_{R}$ or translation $Q_{T}$ between two adjacent equilibrium configurations is small. As a matter of fact, their finite angle rotation around their equilibrium configurations, which are excited thermally or by other means, have been observed recently $[15,16]$. The electrical conductivity between two adjacent flakes has been tuned through their relative, finite angle rotation [17].

Even though an adiabatic translation is presumably dissipationless, the stick-slip motion [18-21] results in a minute energy dissipation and hence small friction coefficient in the sliding of the flake on the monolayer owing to smoothly

\footnotetext{
*ethem.akturk@adu.edu.tr

†ciraci@fen.bilkent.edu.tr
}

varying interaction energy with the small energy barrier involved in the relative translational motion. For this purpose, coating of metal surfaces with graphene is also considered to reduce the sliding friction between them and hence to attain a nearly frictionless sliding regime [22-25]. In addition, owing to the weak interlayer interaction, layered materials were able to be exfoliated by chemical methods [26], laser ablation [27], and by direct charging [28].

Beyond NEMs and nanomechanical devices, the dynamics of a molecule on surfaces of substrates, in particular the coupling of their vibrational and libration modes with the electronic states via charging, the thermal and photoexcitation brought about new insights to fundamental features [29-32]. Also extraordinary properties of graphene flakes of diameter 10-15 $\AA$ attained due to quantum confinement of their electrons in small size are attracting interest for applications in sensors, catalysis, supercapacitors, bioimaging, luminescence, and spintronic devices [33-37].

In this paper, we studied the rotational and translational dynamics of a particular flake on a graphene monolayer. Our objective is to reveal the critical parameters of motion in order to control the dynamics for possible future applications. The attractive interaction between the graphene flake and monolayer (or graphite surface) is size and geometry specific, since it can be modified through the minute chemical interactions at the edge of the flake. The flake becomes bound to the monolayer at a specific equilibrium registry and hence similar flakes may display self-assembly features [38]. We showed that the rotation of a flake around its equilibrium registry gives rise to a periodic variation of interaction energy with a small energy barrier and hence oscillatory variation of torque. Small angular displacement of the flake leads to a harmonic variation of interaction energy and hence to a linear restoring torque. Our work predicts that a whole molecule or flake may function as if a physical pendulum, performing a simple harmonic motion 
(SHM) with shape and size specific libration frequency, in addition to the vibrations of its atoms. Furthermore, we found that this frequency can be monitored by charging of the flake and by perpendicular electric field. Attachment of any molecule to the nanoflake results in a shift of characteristic frequency. Flakes with specific edge geometry or hydrogen decoration [39-41] can also attain permanent electric and magnetic dipole moment. Hence, patterned flakes can engage in forced oscillations with applied electric or magnetic fields, and their electronic and magnetic properties can be tuned also by the electromagnetic waves exiting vibrations. While the energy barrier to the translation of the flake is small, it can be reduced further when the sliding is combined with rotation. This way the friction force can be reduced dramatically.

Briefly, our work presents an analysis of the interaction energy together with forces originating thereof and demonstrates how these forces can be influenced and tuned by external fields, excess charge and mass, configurational changes to exploit them in various chemical application. While our results are obtained from the calculations on a prototype flake, the underlying physics on the nature of interaction, which is unveiled in the present study, will be critical for the understanding of the dynamics of graphene flakes of different size and geometry.

\section{COMPUTATIONAL METHODOLOGY}

The above predictions regarding the rotational and translational dynamics of the flake on graphene ML are obtained from first-principles pseudopotential calculations based on the spin-polarized density functional theory (DFT) within the generalized gradient approximation including van der Waals (vdW) corrections at DFT-D2 level [42], which was found suitable for layered structures in earlier tests [43]. We used projector-augmented wave potentials [44] and the exchange correlation was approximated with the PerdewBurke-Ernzerhof (PBE) functional [45]. The vacuum spacing between adjacent flake+graphene systems is taken to be $20 \AA$ to minimize their coupling. While optimizing atomic positions using the conjugate gradient method (CG), the energy convergence value between two consecutive steps was chosen as $10^{-5} \mathrm{eV}$. A maximum force of $0.01 \mathrm{eV} / \AA$ was allowed on each atom. Calculations were carried out using the VASP software [46]. The Brillouin zone (BZ) corresponding to the primitive unit cell was sampled by a grid of $(7 \times 7 \times 1)$ $\mathbf{k}$ points [47]. Kinetic energy cutoff $\hbar^{2}|\mathbf{k}+\mathbf{G}|^{2} / 2 m$ for the plane-wave basis set is taken as $550 \mathrm{eV}$. The k-points sampling, kinetic energy cutoff, and the vacuum spacing are determined according to extensive convergence tests. A Gaussian type smearing method is used with a smearing width of $0.01 \mathrm{eV}$. High temperature $a b$ initio molecular dynamics (MD) calculations are performed using Verlet algorithm, where Newton's equations are integrated with time steps of $2 \mathrm{fs}$.

\section{FLAKE-GRAPHENE INTERACTION}

In this section we will discuss the interaction energy between the graphene flake and 2D graphene ML based on the calculation of self-consistent field total energy of optimized structures. This interaction energy plays a principal role in the rotational and translational dynamics of the flake on the 2D graphene ML. We now start with the calculation of the interaction energy $E_{i}(x, y, z)$, between two parallel graphene monolayers (or a bilayer) in one of two registries, namely $\mathrm{AA}$ and $\mathrm{AB}$ as described in Figs. 1(a) and 1(b), as a function of their separation $z$. Two parallel monolayers are parallel to the $(x, y)$ plane. The layer binding energies are found to be weak and $19.3 \mathrm{meV} /$ atom and $25.2 \mathrm{meV} /$ atom, respectively. These energies are in fair agreement with previous quantum Monte Carlo calculations [48] and show that van der Waals interactions are taken into account properly [43]. In addition, we will use these calculated binding energies to reveal the effect of edge atoms.

Here we considered a specific flake for a proof of concept, which consists of 24 carbon atoms with $D_{6 h}$ point group symmetry. This flake is found to be stable based on $a b$ initio molecular dynamic calculations performed at $T=1000 \mathrm{~K}$. When twofold carbon atoms at its circumference were saturated by hydrogen atoms, this flake would become coronene molecule. Such a flake or pattern of flakes can be produced experimentally by electron beam lithography and oxygen plasma etching on a monolayer. Pure graphene flakes of $\sim 10-15 \AA$ can be produced through the annealing of graphene oxide monolayers at high temperature.

Two possible equilibrium registries of this 24-atom flake on the graphene ML, namely $\mathrm{AA}$ and $\mathrm{AB}$, are illustrated in Figs. 1(c) and 1(d), respectively. All carbon atoms of the flake are on top of the carbon atoms of the monolayer in the AA registry. In the $\mathrm{AB}$ registry, while three alternating carbon atoms of each hexagon of the flake are above the centers of the adjacent three hexagons below, the remaining three are on top of the carbon atoms of the monolayer. One of these three vectors, $\mathbf{r}_{\mathbf{1}}=\mathrm{a} \widehat{\boldsymbol{y}} / \sqrt{3}, \mathbf{r}_{\mathbf{2}, \mathbf{3}}=\mathrm{a} \widehat{\boldsymbol{x}} / 2 \pm \mathrm{a} \hat{\boldsymbol{y}} / \sqrt{12}$ ( $a$ being the lattice constant of graphene, $a=R_{1,2}$ ) can translate the flake from the AA to the $\mathrm{AB}$ registry. The interactions of the flake with the graphene ML are calculated using supercell geometry, where one flake is placed above each $(7 \times 7)$ supercell of graphene, so that each supercell is composed of $122 \mathrm{C}$ atoms. The interaction energy between the flake and monolayer, $E_{i}(x, y, z)$, which normally depends on the lateral position $(x, y)$ and height of the flake $z$, can be cast in the form of $E_{i}(z, \theta)$ for each registry, where $\theta$ is the angular displacement around the equilibrium and $z$ is the separation between the flake and the monolayer.

The binding energy of the flake to the monolayer is calculated to be $1.25 \mathrm{eV}$ and $1.23 \mathrm{eV}$ for AA and AB registry, respectively. Note that in the above discussion the (2D) bilayer at the $A B$ registry was more favorable energetically than the AA registry. However, for a finite size flake the size and symmetry of the flake determines which registry will be more favorable energetically. Comparing with the calculated binding energies of a bilayer having $\mathrm{AA}$ and $\mathrm{AB}$ registries given above, one concludes that the weak chemical interaction between two-folded carbon atoms at the edge of the bare flake and the monolayer contributes to the above total binding energy by $66 \mathrm{meV} /$ atom and $60 \mathrm{meV} /$ atom, respectively. Then, one can argue that the interaction between a flake and the graphene ML, and hence the parameters of the dynamics, is expected to be dominated by twofold coordinated bare carbon edge atoms. 
(a)

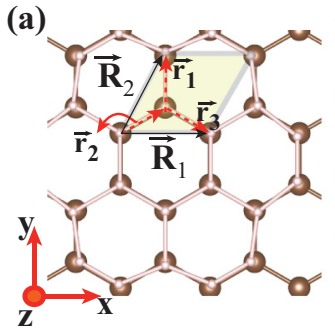

AA-Registry

(c)

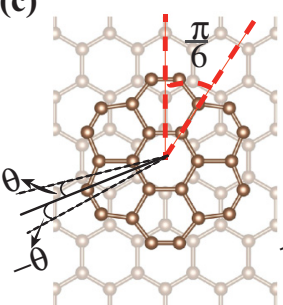

$\omega=5.24 \times 10^{12} \mathrm{rad} . \mathrm{s}^{-1}$

$f=0.83 \mathrm{THz}$ (b)

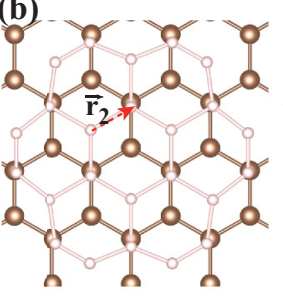

AB-Registry

(d)

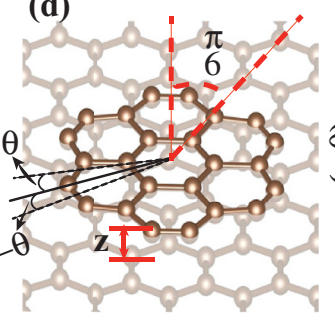

$\omega=2.54 \times 10^{12} \mathrm{rad} . \mathrm{s}^{-1}$

$f=0.40 \mathrm{THz}$ (e)
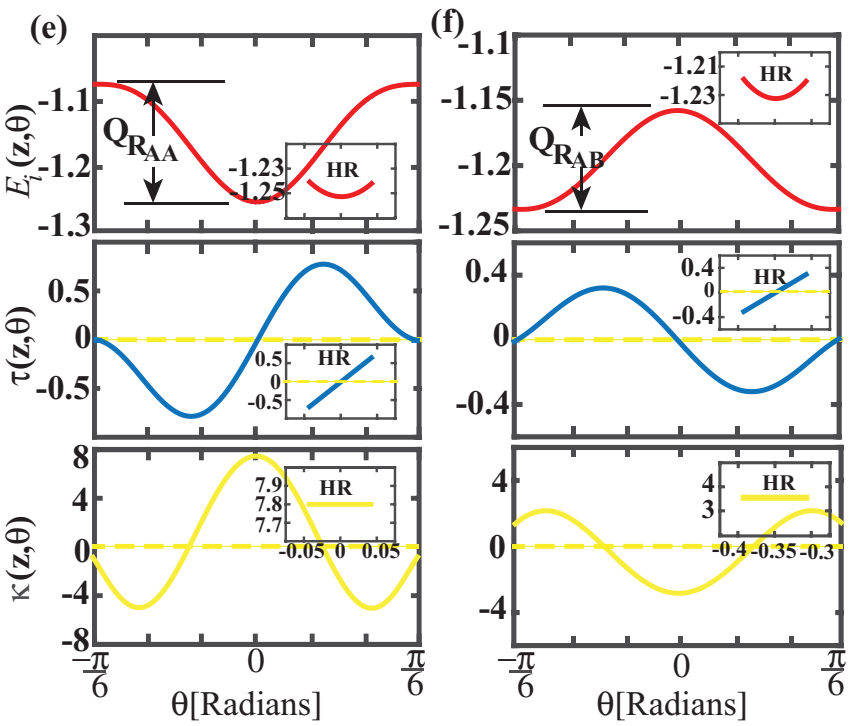

FIG. 1. (a) and (b) Atomic configurations of $\mathrm{AA}$ and $\mathrm{AB}$ registries of a graphene bilayer with the primitive unit cell and lattice translation vectors, $\mathbf{R}_{\mathbf{1}}$ and $\mathbf{R}_{\mathbf{2}}$. Three translation vectors between these two registries are $\mathbf{r}_{i}(i=1-3)$. (c) and (d) Top and tilted views of the atomic configuration of the graphene flake+monolayer system in $\mathrm{AA}$ and $\mathrm{AB}$ registries. Their calculated characteristic frequencies are also given. (e) The variation of interaction energy $E_{i}(z, \theta)$ (in eV) having the barrier of $Q_{R_{A A}}=180 \mathrm{meV}$ between two adjacent minima, torque $\tau(z, \theta)$ (in $\mathrm{eV} / \mathrm{rad}$ ) and angular force constant $\kappa(z, \theta)$ (in $\mathrm{eV} / \mathrm{rad}^{2}$ ) for $-\pi / 6<\theta<\pi / 6$ at the AA registry. (f) The variation of $E_{i}(z, \theta)$ with $Q_{R_{A B}}=75 \mathrm{meV}$ between two adjacent minima, $\tau(z, \theta)$ and $\kappa(z, \theta)$ for $-\pi / 6<\theta<\pi / 6$ at the AB registry. The harmonic parts of $E_{i}$ corresponding to small angular displacement, linear restoring torque $\tau$, and constant $\kappa$ are shown by insets for both registries.

\section{Effect of hydrogen saturation}

We next address the following question: How the interaction energy and forces can undergo changes when the bare and low coordinated atoms of the flake in Figs. 1(c) and 1(d) are saturated by 12 hydrogen atoms to form the coronene molecule? To this end, we calculated the minimum binding energy of the coronene molecule to the graphene ML. Interestingly, the equilibrium configuration changed from AA registry to $\mathrm{AB}$ registry by going from the bare flake to the coronene molecule. Moreover, the total binding energies are found to be $1.44 \mathrm{eV}$ and $1.24 \mathrm{eV}$ for $\mathrm{AB}$ and $\mathrm{AA}$ registries, respectively, which are stronger than those energies calculated for the bare flake. Such a situation was pointed out also for other molecules [49]. We explain this paradoxical situation as follows: While twofolded carbon atoms at the edge have relatively smaller chemical interaction upon saturation with hydrogen atoms, the vdW interaction of these 12 hydrogen atoms of the coronene molecule compensates the reduction of the chemical interaction to result in a slightly stronger binding for both registries. The recent work by Peymanirad et al. [9] found the same $\mathrm{AB}$ registry for the coronene molecule. Moreover, they calculated the energy difference between $\mathrm{AA}$ and $\mathrm{AB}$ registries as $8.3 \mathrm{meV} /$ atom. This is in good agreement with the present study finding the same energy difference as $8.1 \mathrm{meV} /$ atom. It is, therefore, corroborated that the binding and resulting parameters of motion of a flake can undergo changes upon saturation of edge atoms by adatoms like hydrogen or oxygen. We note that there are symmetric bare graphene flakes comprising a diverse number of carbon atoms, and also the corresponding molecules with all carbon atoms at the edges are saturated with hydrogens. Also there are experiments carried out with bare flakes.

It should be noted that the interaction between a flake and the graphene ML and resulting frequencies related with its rotational dynamics, which will be calculated in the forthcoming sections, are dependent on the size and geometry. Triangular, hexagonal flakes, or flakes with any special geometry and size could have been treated and for each flake different values of interaction energy and frequencies would be attained. However, the physics underlying the interaction between a flake and the graphene ML is unaltered. In this study, rather than considering flakes of different size and geometry and engaging large scale computations, we carried out our analysis using a specific bare flake as a proof of concept and examined the nature of interaction and revealed the underlying physics. We then investigated how to control its dynamics externally. To this end, we examined the effect of charging, applied perpendicular and lateral electric field, a small molecule attached to the flake etc. in the forthcoming sections.

\section{ROTATIONAL DYNAMICS}

The variation of $E_{i}(z, \theta)$ shown in Fig. 1(e) is calculated from the fully relaxed geometry and has its minimum at $\theta=0$ at the AA registry. The rotational barrier $Q_{R_{A A}}=180 \mathrm{meV}$. The variation of the torque $\tau(z, \theta)=-\partial E_{i}(z, \theta) / \partial \theta$ and force constant $\kappa(z, \theta)=\partial^{2} E_{i}(z, \theta) / \partial^{2} \theta$ are also plotted. For small angular displacements around the minimum of $E_{i}(z, \theta)$, the restoring torque tends to maintain the equilibrium position of the flake at $\theta=0$ at the AA registry [but $\theta=0.35$ radians 
or $20.1^{\circ}$ at the $\mathrm{AB}$ registry as shown in Fig. 1(f)]. Since the interaction energy is harmonic for small $\theta, \kappa$ is constant as indicated in Fig. 1(e) and hence the flake can execute a simple harmonic motion (SHM). Similar curves are obtained for AB registry in Fig. 1(f) with rotational barrier, $Q_{R_{A B}}=75 \mathrm{meV}$. Since the AA registry is rather symmetric, we will continue our analysis of the dynamics of the flake for this registry.

Using the moment of inertia $I$, the libration frequency of the bare flake is calculated as $f=(1 / 2 \pi) \sqrt{\kappa / I}=0.83 \mathrm{THz}$ (or $\omega=5.24 \times 10^{12} \mathrm{rad} / \mathrm{s}$ ) for AA registry. It is higher than usual libration frequencies of big molecules due to the increased interaction at the edges with monolayer. In the quantum limit, the libration energy corresponding to $n$th level excitation is $E_{\ell, n}=(n+1 / 2) \hbar \omega$ with the phonon energy $\hbar \omega=3.4$ $\mathrm{meV}$. Clearly, the libration frequency of the flake is size and shape specific and anharmonic effects become crucial at high temperature. As one expects, the libration frequency at the $\mathrm{AB}$ registry, $f=0.40 \mathrm{THz}$, is smaller than that at the AA registry. It is noted that the interaction potential and hence the frequency of oscillation is modified depending on whether the underlying monolayer is accelerated or decelerated.

More recently, Wang et al. reported the thermally induced rotation of graphene flake on $\mathrm{h}-\mathrm{BN}$ monolayer around the equilibrium registry [15]. They observed that flakes of $\sim 250 \mathrm{~nm} \times$ $500 \mathrm{~nm}$ size can rotate by $\pi / 6$ from their equilibrium for $T>100{ }^{\circ} \mathrm{C}$. Despite the fact that the symmetric graphene flake in the present study is relatively smaller and is placed on the graphene substrate, this experiment justifies our model. Here the rotation of the flake can necessitate higher temperature, even if it may be lowered due to thermal fluctuations or quantum tunneling [50].

\section{A. Effect of adsorbed molecules and adatoms}

The frequency of the flake can shift due to a molecule, which is anchored to it. As a proof of concept, we considered the neutral glycine $\mathrm{C}_{2} \mathrm{H}_{5} \mathrm{NO}_{2}$, which is chemisorbed from its nitrogen atom to the flake as shown in Fig. 2(a). Our intention here is to demonstrate that the libration frequency decreases upon the attachment of a molecule on the flake. The coverage of the flake by foreign adatoms like $\mathrm{H}, \mathrm{O}, \mathrm{Cl}, \mathrm{F}$ and transition metal atoms such as Ti, Mo, Co. Even if the change of weight due to the adsorbed molecule and hence resulting small normal force is not counted (in principle, it can easily be counted self-consistently by decreasing $z$ and eventually by generating a constant normal force equal to the weight of the molecule) $E_{i}$ is still affected due to the absorbed molecule and possible local disturbance of electronic structure induced by the adsorbate [51]. Additionally, the moment of inertia also increases to reduce the frequency. Since the frequency shifts can be measured by precision, they can be taken as fingerprints of a molecule, which is adsorbed at well-defined locations on a specific flake. Hence, these physical effects can be utilized to develop a nanosensor.

\section{B. Effect of charging}

The libration frequency of the flake can be monitored by charging it with excess electrons as described in Fig. 2(b), whereby part of the charge is transferred to the flake; the (a)
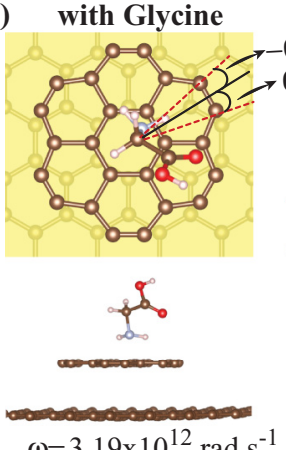

$\omega=3.19 \times 10^{12} \mathrm{rad} . \mathrm{s}^{-1}$

(c)

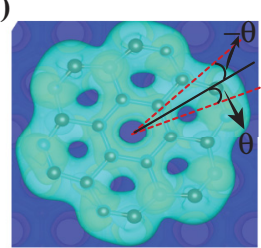
$f=0.51 \mathrm{THz}$ (b)

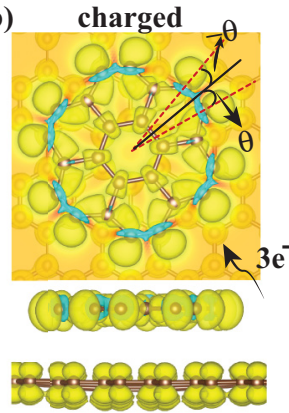

$\omega=2.53 \times 10^{12} \mathrm{rad} . \mathrm{s}^{-1}$

$f=0.40 \mathrm{THz}$

E-field

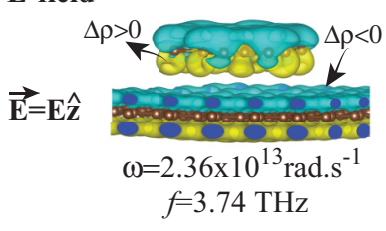

FIG. 2. Tuning the dynamics of rotational motion of a graphene flake on the graphene ML: (a) Calculated characteristic frequency of simple harmonic motion ( $\mathrm{SHM}$ ) of glycine $\mathrm{C}_{2} \mathrm{H}_{5} \mathrm{NO}_{2}$ anchored to the flake. (b) Isosurfaces of $\Delta \rho$ (see text) of the flake+monolayer graphene system charged by three electrons per supercell and its calculated frequency. (c) Top and side views of SHM of the flake under the perpendicular electric field $\mathbf{E}_{\perp}$ of $1 \mathrm{~V} / \AA$ along the $z$ direction and its calculated characteristic frequency. Isosurfaces of $\Delta \rho$ indicates polarization induced by $\mathbf{E}_{\perp}$. Turquoise regions indicate electron depletion. Angular frequency $\omega$ or frequency $f$ of the bare flake has changed in (a), (b), and (c).

remaining part is distributed in the monolayer to make it metallic. Here, the excess electron density is obtained as the difference of the total densities of occupied states of the charged $(c)$ and bare $(b)$ flake+monolayer system, namely $\Delta \rho(\mathbf{r})=\rho_{c, T}(\mathbf{r})-\rho_{b, T}(\mathbf{r})$. This way, the Coulomb repulsion developed between monolayer and flake [28] weakens the interaction energy and hence causes the frequency of bare flake to change. In the present study, we calculated that the frequency of the bare flake is reduced from $0.83 \mathrm{THz}$ to $0.40 \mathrm{THz}$ under the charging of three electrons per supercell. A polarity between the flake and the monolayer due to charge rearrangements and charge transfer between them can also be generated by the external electric field $E_{\perp}$ as shown in Fig. 2(c). Polarization induces attractive force between the flake and SL and hence enhances the interaction between them. At the same time holes in the flake and excess electrons are generated in the monolayer as discussed in the forthcoming part. Under these circumstances, the frequency attains significant blueshifts. Note that calculated frequency shifts and electronic charge rearrangements in Fig. 2 are consistent.

\section{Effect of electric and magnetic field}

In contrast to the perpendicular electric field, a lateral field $E_{\|}$can induce an electric dipole between the opposite edges of the flake. This dipole by itself is aligned with the 

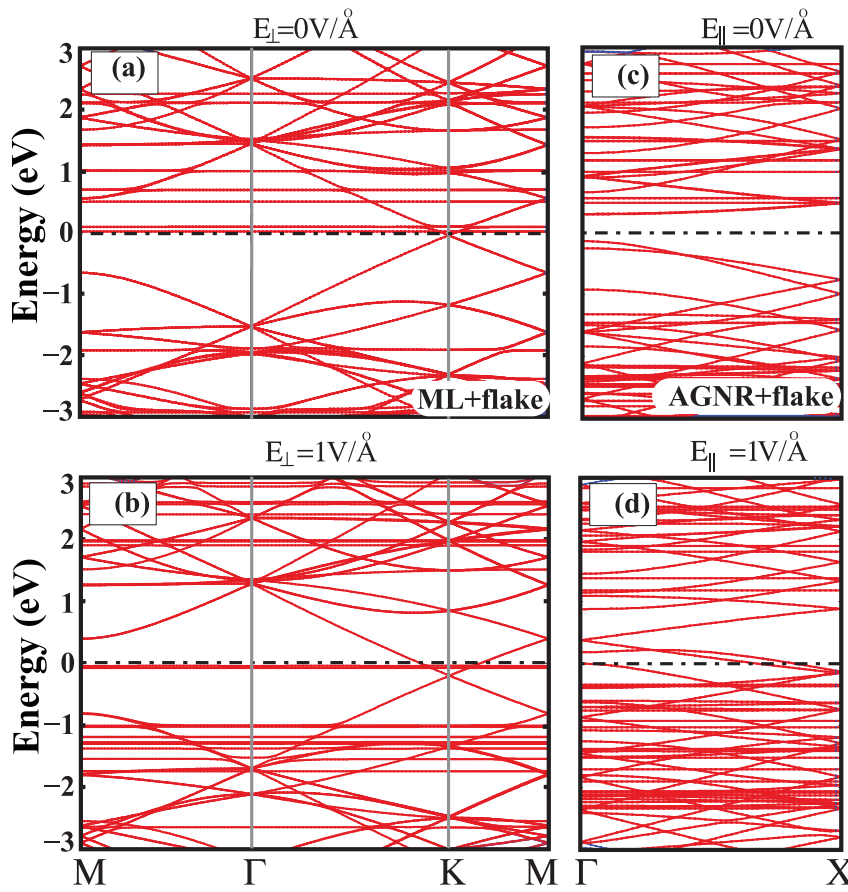

FIG. 3. Tuning of the electronic structure of the flake+monolayer system by perpendicular $E_{\perp}$, and lateral $E_{\|}$, electric field: (a) The electronic structure of the flake+monolayer corresponding to $\theta=0$ and $\mathbf{E}=0$. (b) The electronic structure in (a) is tuned by applying $E_{\perp}=1.0 \mathrm{~V} / \AA$ at $\theta=0$. (c) The electronic structure of the flake on an armchair nanoribbon for $\theta=0$ and $\mathbf{E}=0$. (d) The electronic structure (c) is tuned at $\theta=0$ by applying $E_{\|}=1.0 \mathrm{~V} / \AA$. Bands are calculated by using the local basis set.

applied lateral electric field to minimize its potential energy. Accordingly, one can rotate the flake by rotating the field; even the forced oscillations of the flake can be induced. Similar to a nanocompass, the flake of specific geometry like a piece of nanoribbon having a permanent magnetic moment [39-41] can be rotated by an external magnetic field. It should be noted that in the calculations involving charging and/or external electric field, we used a local basis set [52] to circumvent the escape of the excess charge to the vacuum region $[53,54]$ occurring as an artifact of the plane wave calculations within periodic boundary conditions.

Once the flake is rotated by an angle $\theta$ from its equilibrium configuration relative to the monolayer, the electronic structure of the flake+monolayer system can be modified. While normally the interaction between three folded carbon atoms in the nanoflake and monolayer is weak, it is slightly enhanced due to $p_{z}-p_{z}$ bonding states formed when these carbon atoms become on top each other upon rotation [55]. This way the electrical conductivity of the flake+SL can be enhanced [17]. Even if the change in the electronic structure attained by the angular displacement can be weak owing to large $z$ and hence weak interaction between the flake and the monolayer, it can nevertheless be enhanced by the perpendicular electric field. The electronic structure of the flake+monolayer and the flake+nanoribbon systems are calculated under the applied electric field $E_{\perp}$ and $E_{\|}$, respectively, for $\theta=0$. In Fig. 3(a), the electronic structure of the flake+graphene under $E_{\perp}=0$

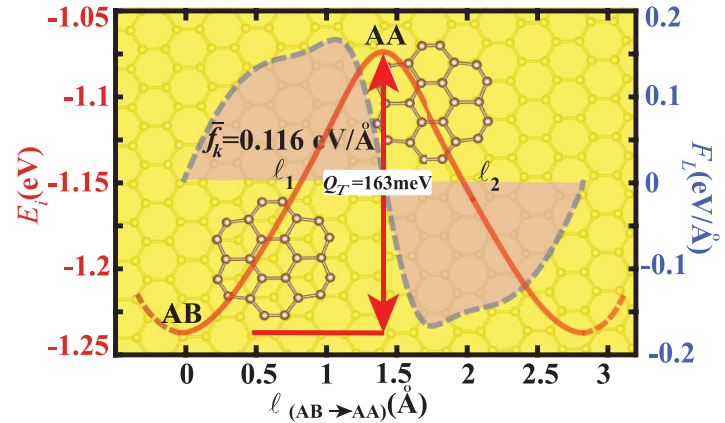

FIG. 4. Translation of a graphene flake on the graphene ML: Atomic configuration of the flake translating (sliding) from the minimum energy $\mathrm{AB}$ configuration to a maximum energy configuration AA (shown in the background). Variation of the total energy (shown by solid line) in the course of sliding from $\mathrm{AB} \rightarrow \mathrm{AA} \rightarrow \mathrm{AB}$. The energy barrier is $Q_{T}=163 \mathrm{meV}$. Variation of the lateral force, $F_{L}$ (shown by dashed line) in the course of translation of the flake.

in equilibrium can be viewed as the superposition of the bare graphene ML and the single flake with flat bands in $(7 \times 7)$ supercell showing a minute chemical interaction. The Dirac cone at the $K$ point determines the Fermi level and indicates almost no charge transfer between flake and monolayer. In Fig. 3(b), under $E_{\perp}=1.0 \mathrm{eV} / \AA$ along the $z$ direction, the transfer of 0.13 electrons from the flake results in the metallization of the monolayer with downshift of the Dirac cone. The energies of the electrons in the flake are eventually raised by $\sim 2 \mathrm{eV}$ relative to the bands of the monolayer despite their small downshift due to the charge depletion. Flat bands of the flake just below $E_{F}$ are raised from lower energies and pin the Fermi level.

A lateral electric field applied to the 2D supercell of the flake+graphene monolayer causes divergences. Therefore, we consider the flake placed on a graphene armchair nanoribbon having vacuum spacing on both sides. The lateral electric field $E_{\|}$is perpendicular to the axis of the ribbon. As seen in Fig. 3(c), the flake+nanoribbon is normally a semiconductor of direct band gap of $\sim 0.4 \mathrm{eV}$. However, under $E_{\|}=1.0 \mathrm{eV}$, bands are shifted and tilted, and consequently not only the nanoribbon but also the flake becomes metal as shown in Fig. 3(d). This way perpendicular and lateral electric fields exerting concomitantly or individually can be used to tune the electronic structure of the flake+graphene system locally. If many flakes assembled on a monolayer, their electronic structure and hence their optical properties can be graded in direct space; even they can form an optical lattice through the monitoring of $E_{\perp}$ and $E_{\|}$.

\section{TRANSLATIONAL DYNAMICS}

We next consider the dynamics related with the translation (or sliding) of the flake on the monolayer. As shown in Fig. 4, the variation of the attractive interaction energy $E_{i}(x, y, z)$, where $x$ and $y$ trace the displacement of $\mathrm{AB} \rightarrow \mathrm{AA} \rightarrow \mathrm{AB}$ (equivalent to the displacement along $\mathbf{r}_{1}+\mathbf{r}_{2}$ ) is calculated by optimizing $z$ and the positions of other atoms. It is periodic and involves a small translational energy barrier, $Q_{T}=163 \mathrm{meV}$. The lateral force variation is calculated from the expression 
$F_{L}=-\partial E_{i} / \partial \ell$, where $\partial \ell$ is infinitesimal translation along $\mathbf{r}_{1}$ or $\mathbf{r}_{2}$. Ideally, in the adiabatic translation of one period, the net work done by the lateral force is $\int F_{L} d \ell=0$ and hence the mechanical energy is not dissipated. In reality, the mechanical energy, which is stored in the translation, $\mathrm{AB} \rightarrow \mathrm{AA}$, cannot be gained back in the sudden (or nonadiabatic) translation from $\mathrm{AA} \rightarrow \mathrm{AB}$, but it is dissipated. This gives rise to the stick-slip motion [18-21,25,56]. In the course of the sudden translation between $\mathrm{AA}$ and $\mathrm{AB}$ the velocities of flake and monolayer atoms exceed the center of mass velocity sometimes by orders of magnitude [57]; the excited local vibrations evolve into nonequilibrium phonons via anharmonic couplings [58]. In Fig. 4 the average kinetic friction force calculated as $\bar{f}_{k}=$ $\int_{A B}^{A A} F_{L} d \ell /\left|\mathbf{r}_{1}\right|$, which can be the upper bound for the average kinetic friction force in the sliding through $\mathbf{r}_{1}+\mathbf{r}_{2}$. Clearly it is small enough to let us to consider lubricants made from flakes. We found that $Q_{T}$ can decrease even diminish to lead to frictionless sliding, if the translation of flake is combined by the rotation, whereby the energy barrier is reduced. Also combined with aforementioned results related with charging the energy dissipation has to decrease under charging but has to increase under the applied electric field perpendicular to the flake+monolayer system.

\section{CONCLUSION}

In conclusion, the interaction between a graphene nanoflake and graphene ML is weak, but it can set a well defined registry and restoring force against rotational and linear displacements. This way, similar flakes can be self-assembled and can operate as an ensemble of physical pendulums. As a proof of concept, we demonstrated that external charging, changes of atomic configurations induced by rotation, externally applied perpendicular or lateral magnetic and electric fields can influence the parameters of motion of the flake in its rotational and translational dynamics on the monolayer. As a result, the libration frequency, electronic and magnetic properties, and electrical conductivity of the nanoflake or molecule are modified. The rotational and translational dynamics can also be affected by the adsorption or physisorption of adatoms or molecules. This way, the mass of the flake due to a molecule anchored on it increases, which in turn changes the dynamics. Specific adatoms can also modify the interaction energy between the nanoflake and monolayer. Additionally, the nanoflake can be functionalized by adatoms or molecules and they attain permanent magnetic dipole moments, which destroy the spin degeneracy at the Fermi level. In this respect, the nanoflake+monolayer system can be utilized as spin valves or sensors.

In the sliding of flakes, the lateral force to translate the flake is weak and oscillatory; it is dissipationless in the adiabatic sliding but normally proceeds as stick-slip motion with small friction coefficient. The energy barrier in the translation of flake can even diminish by rotating or charging it in the course of sliding. Additionally, similar flake-monolayer pairs of different materials, like h-BN, h-MoS 2 , silicene, and phosphorene etc. can display marked physical properties and initiate an original concept in the design of nanocomposite materials.

\section{ACKNOWLEDGMENTS}

The computational resources are provided by TÜBITAK ULAKBIM, High Performance and Grid Computing Center (TR-Grid e-Infrastructure). The authors of the paper acknowledge financial support from the Academy of Sciences of Turkey TÜBA. H.H.G. acknowledges the support of TÜBITTAK under Grant No. 114F453.
[1] A. K. Geim and I. V. Griogorieva, Nature (London) 499, 419 (2013).

[2] R. A. van Delden, M. K. J. ter Wiel, M. M. Pollard, J. Vicario, N. Koumura, and B. L. Feringa, Nature (London) 437, 1337 (2005).

[3] Y. Shiria, A. J. Osgood, Y. Zhao, Y. Yao, L. Saudan, H. Yang, C. Y. Hung, L. B. Alemany, T. Sasaki, J.-F. Morin, J. M. Guerrerero, K. F. Kelly, and J. M. Tour, J. Am. Chem. Soc. 128, 4854 (2006).

[4] S. Khatua, J. M. Guerrero, K. Claytor, G. Vives, A. B. Kolomeisky, J. M. Tour, and S. Link, ACS Nano 3, 351 (2009).

[5] M. Schliwa and G. Woehlike, Nature (London) 422, 759 (2003).

[6] G. S. Kottas, L. I. Clarke, D. Horinek, and J. Michi, Chem. Rev. 105, 1281 (2005).

[7] R. Bistritzer and A. H. MacDonald, Proc. Natl. Acad. Sci. (USA) 108, 12233 (2011).

[8] M. Yankowitz, J. Xue, D. Cormode, J. D. Sanchez-Yamagishi, K. Watanabe, T. Taniguchi, P. Jarillo-Herrero, P. Jacquod, and B. J. LeRoy, Nat. Phys. 8, 382 (2012).

[9] F. Peymanirad, S. K. Singh, H. Ghorbanfekr-Kalashami, K. S. Novoselov, and F. M. Peters, 2D Mater. 4, 025015 (2017).
[10] D. M. Eigler, C. P. Lutz, and W. E. Rudge, Nature (London) 352, 600 (1991).

[11] C. Manzano, W. H. Soe, H. S. Wong, F. Ample, A. Gourdon, N. Chandrasekhar, and C. Joachim, Nat. Mater. 8, 576 (2009).

[12] U. G. E. Perera, F. Ample, H. Kersell, Y. Zhang, G. Vives, J. Echeverria, M. Grisolia, G. Rapenne, C. Joachim, and S.-W. Hla, Nat. Nanotechnol. 8, 46 (2013).

[13] M. Koshina, N. Solin, T. Tanaka, H. Isobe, and E. Nakamura, Nat. Nanotechnol. 3, 595 (2008).

[14] J. H. Warner, Y. Ito, M. Zaka, 1. Ge, T. Akachi, H. Okimoto, K. Porfyrakis, A. A. R. Watt, H. Shinohara, and G. A. D. Briggs, Nano Lett. 8, 2328 (2008).

[15] D. Wang, G. Chen, C. Li, M. Cheng, W. Yang, S. Wu, G. Xie, J. Zhang, J. Zhao, X. Lu, P. Chen, G. Wang, J. Meng, J. Tang, R. Yang, C. He, D. Liu, D. Shi, K. Watanabe, T. Taniguchi, J. Feng, Y. Zhang, and G. Zhang, Phys. Rev. Lett. 116, 126101 (2016).

[16] J. Choe, Y. Lee, L. Fang, G.-D. Lee, Z. Bao, and K. Kim, Nanoscale 8, 13174 (2016).

[17] E. Koren, I. Leven, E. Lörtscher, A. Knoll, O. Hod, and U. Duerig, Nat. Nanotechnol. 11, 752 (2016).

[18] L. Prandtl, Z. Angew. Math. Mech. 8, 85 (1928).

[19] G. A. Tomlinson, Philos. Mag. 7, 905 (1929). 
[20] C. M. Mate, G. M. McClelland, R. Erlandsson, and S. Chiang, Phys. Rev. Lett. 59, 1942 (1987).

[21] A. Buldum and S. Ciraci, Phys. Rev. B 55, 2606 (1997).

[22] A. E. Filippov, M. Dienwiebel, J. W. M. Frenken, J. Klafter, and M. Urbakh, Phys. Rev. Lett. 100, 046102 (2008).

[23] T. Filleter, J. L. McChesney, A. Bostwick, E. Rotenberg, K. V. Emtsev, T. Seyller, K. Horn, and R. Bennewitz, Phys. Rev. Lett. 102, 086102 (2009).

[24] C. Lee, Q. Li, W. Kalb, X-Z. Liu, H. Berger, R. W. Carpick, and J. Hone, Science 328, 76 (2010).

[25] S. Cahangirov, C. Ataca, M. Topsakal, H. Sahin, and S. Ciraci, Phys. Rev. Lett. 108, 126103 (2012); S. Cahangirov, S. Ciraci, and V. O. Ozcelik, Phys. Rev. B 87, 205428 (2013).

[26] H. C. Sniepp, J. L. Li, M. J. McAllister, H. Sai, M. HerreraAlonso, D. H. Adamson, R. K. Prud'homme, R. Car, D. A. Saville, and I. A. Aksay, J. Phys. Chem. B 110, 8535 (2006).

[27] F. Carbone, P. Baum, P. Rudolf, and A. H. Zewail, Phys. Rev. Lett. 100, 035501 (2008); Y. Miyamoto, H. Zhang, and D. Tomanek, ibid. 104, 208302 (2010).

[28] M. Topsakal, and S. Ciraci, Appl. Phys. Lett. 98, 131908 (2011).

[29] M. Wahl, M. Stör, H. Spillmann, T. A. Jung, and L. H. Gade, Chem. Commun. 1349 (2007).

[30] J. Schaffert, M. C. Cottin, A. Sonntag, and H. Karacuban, C. A. Bobisch, N. Lorente, J.-P. Gauyacq, and R. Möller Nat. Mater. 12, 223 (2013).

[31] T. Ishikawa, S. A. Hayes, S. Keskin, G. Corthey, M. Hada, K. Pichugin, A. Marx, J. Hirscht, K. Shionuma, K. Onda, Y. Okimoto, S.-y. Koshihara, T. Yamamoto, H. Cui, M. Nomura, Y. Oshima, M. Abdel-Jawad, R. Kato, and R. J. D. Miller, Science 350, 1501 (2015).

[32] Y. Tong, T. Kampfrath, and R. K. Campen, Phys. Chem. Chem. Phys. 18, 18424 (2016).

[33] W. L. Wang, S. Meng, and E. Kaxiras, Nano Lett. 8, 241 (2008); W. L. Wang, O. V. Yazyev, S. Meng, and E. Kaxiras, Phys. Rev. Lett. 102, 157201 (2009).

[34] H. Sahin, R. T. Senger, and S. Ciraci, J. Appl. Phys. 108, 074301 (2010).

[35] L. S. Li and Y. Yan, J. Phys. Chem. Lett. 1, 2572 (2010).

[36] L. Li, G. Wu, G. Yang, J. Peng, J. Zhao, and J. J. Zu, Nanoscale 5, 4015 (2013).
[37] X. Wang, G. Sun, N. Li, and P. Chen, Chem. Soc. Rev. 45, 2239 (2016).

[38] F.-J. M. Heringdorf, M. C. Reuter, and R. M. Tromp, Nature (London) 412, 517 (2001).

[39] E. H. Lieb, Phys. Rev. Lett. 62, 1201 (1989).

[40] M. Topsakal, E. Akturk, H. Sevincli, and S. Ciraci, Phys. Rev. B 78, 235435 (2008).

[41] H. Sahin, C. Ataca, and S. Ciraci, Appl. Phys. Lett. 95, 222510 (2009).

[42] S. Grimme, J. Comput. Chem. 27, 1787 (2006).

[43] C. Ataca, M. Topsakal, E. Akturk, and S. Ciraci, J. Phys. Chem. C 115, 16354 (2011).

[44] P. E. Blöchl, Phys. Rev. B 50, 17953 (1994).

[45] J. P. Perdew, K. Burke, and M. Ernzerhof, Phys. Rev. Lett. 77, 3865 (1996).

[46] G. Kresse and J. Furthmüller, Phys. Rev. B 54, 11169 (1996).

[47] H. J. Monkhorst and J. D. Pack, Phys. Rev. B 13, 5188 (1976).

[48] E. Mostaani, N. D. Drummond, and V. I. Falko, Phys. Rev. Lett. 115, 115501 (2015).

[49] J. Björk, F. Hanke, C.-A. Palma, P. Samori, M. Cecchini, and M. Persson, J. Phys. Chem. Lett. 1, 3407 (2010).

[50] R. M. Dimeo, Am. J. Phys. 71, 885 (2003).

[51] E. Tekman and S. Ciraci, Phys. Rev. B 40, 10286 (1989); S. Ciraci, A. Baratoff, and I. P. Batra, ibid. 42, 7618 (1990).

[52] J. M. Soler, E. Artacho, J. D. Gale, A. Garcia, J. Junquera, P. Ordejon, and D. Sanchez-Portal, J. Phys.: Condens. Matter 14, 2745 (2002).

[53] M. Topsakal and S. Ciraci, Phys. Rev. B 85, 045121 (2012).

[54] M. Topsakal, H. H. Gürel, and S. Ciraci, J. Phys. Chem. C 117, 5943 (2013).

[55] I. P. Batra, N. García, H. Rohrer, H. Salemink, E. Stoll, and S. Ciraci, Surf. Sci. 181, 126 (1987).

[56] W. Zhong, and D. Tománek, Phys. Rev. Lett. 64, 3054 (1990); D. Tománek, W. Zhong, and H. Thomas, Europhys. Lett. 15, 887 (1991).

[57] M. H. Müser, M. Urbakh, and M. O. Robbins, Adv. Chem. Phys. 126, 187 (2003).

[58] V. L. Gurevich, Transport in Phonon Systems (Elsevier Science Publishers, North-Holland, Amsterdam, 1986). 